\title{
A tecnologia como ferramenta de ensino-aprendizagem em formações na área de saúde: uma revisão bibliográfica
}

\author{
Technology as a teaching-learning tool in healthcare education: a literature review
}

La tecnología como herramienta de enseñanza-aprendizaje en la educación en salud: revisión de la

\section{literatura}

\section{Resumo}

O estudo da disciplina de anatomia exige do aluno alta capacidade de atenção e manutenção da concentração para o alcance do aprendizado em sua totalidade, tornando-se um processo laborioso e extenuante devido à sua intrincada riqueza de detalhes. Tal fato corrobora para a dispersão da atenção do aluno e o fatigante empenho do docente em tentar prender a atenção dos mesmos durante o processo de ensino-aprendizagem. Dessa forma, ferramentas tecnológicas visam ampliar as possibilidades das práticas pedagógicas contribuindo para o maior envolvimento do discente ao atribuir a autonomia do processo de aprendizagem. Na modernidade, novas tecnologias são implementadas como auxiliadoras da sala de aula, pois permitem o enriquecimento do conteúdo aplicado pelo professor aos alunos. O presente artigo tem como objetivo averiguar a existência de evidências científicas relacionadas ao uso da tecnologia para o ensino da anatomia através da busca ativa em plataformas de pesquisa científica. Foram encontrados 180 artigos relacionados com as palavras-chave selecionadas e dispostos em quadros de análise entre os anos de 2008 a 2018, nas línguas inglês, português e espanhol. Destes, 9,4\% artigos foram selecionados para a leitura integral dos seus respectivos resumos e deste; $41,17 \%$ foram selecionados para a leitura integral. Os principais temas encontrados foram a interferência da tecnologia do ensino da disciplina de anatomia, a comparação entre o método tradicional de ensino e a metodologia associada ao uso de realidade virtual e simulação e a motivação dos alunos diante da possibilidade do uso de novas tecnologias em processos de ensino-aprendizagem. Os resultados demonstraram que existem fortes evidências de que o uso de recursos tecnológicos estreita as relações de alunos e professores, uma vez que o processo se torna mais lúdico e integrativo ao passo que torna o aluno autônomo de seu próprio direcionamento em sala de aula, reduzindo a exaustão inerente ao estudo da disciplina em seu modo tradicional.

Palavras-chave: Tecnologia; Ensino; Anatomia; Bioengenharia.

\section{Abstract}

The study of the anatomy discipline requires from the student a high capacity for attention and maintenance of concentration to achieve learning in its entirety, making it a laborious and exhausting process due to its intricate wealth of details. This fact corroborates the dispersion of student attention and the tiring effort of the teacher in trying to hold their attention during the teaching-learning process. Thus, technological tools aim to expand the possibilities 
of pedagogical practices contributing to greater student involvement by attributing the autonomy of the learning process. Nowadays, new technologies are implemented as helpers in the classroom, as they allow the enrichment of the content applied by the teacher to the students. This article aims to investigate the existence of scientific evidence related to the use of technology for teaching anatomy through active search in scientific research platforms. 180 scientific articles were found related to the selected keywords and arranged in analysis tables between the years 2008 to 2018, in English, Portuguese and Spanish. Of these, 9.4\% articles were selected for full reading of their respective abstracts and this one; $41.17 \%$ were selected for full reading. The main themes found were the interference of technology in teaching the discipline of anatomy, the comparison between the traditional teaching method and the methodology associated with the use of virtual reality and simulation, and the motivation of students regarding the possibility of using new technologies in processes of teaching-learning. The results showed that there is strong evidence that the use of technological resources narrows the relationships of students and teachers, since the process becomes more playful and integrative while making the student autonomous from his own direction in the classroom, reducing the exhaustion inherent in studying the discipline in its traditional way.

Keywords: Technology; Teaching; Anatomy; Bioengineering.

\section{Resumen}

El estudio de la disciplina de anatomía requiere del alumno una alta capacidad de atención y mantenimiento de la concentración para lograr el aprendizaje en su totalidad, convirtiéndolo en un proceso laborioso y agotador debido a su intrincada riqueza de detalles. Este hecho corrobora la dispersión de la atención del alumno y el agotador esfuerzo del docente por intentar retener su atención durante el proceso de enseñanza-aprendizaje. Así, las herramientas tecnológicas tienen como objetivo ampliar las posibilidades de las prácticas pedagógicas contribuyendo a una mayor implicación del alumno atribuyéndole la autonomía del proceso de aprendizaje. En la modernidad, las nuevas tecnologías se implementan como ayudantes en el aula, ya que permiten el enriquecimiento de los contenidos aplicados por el docente a los alumnos. Este artículo tiene como objetivo investigar la existencia de evidencia científica relacionada con el uso de la tecnología para la enseñanza de la anatomía a través de la búsqueda activa en plataformas de investigación científica. Se encontraron 180 artículos relacionados con las palabras clave seleccionadas y ordenados en tablas de análisis entre los años 2008 a 2018, en inglés, portugués y español. De estos, el 9,4\% de los artículos fueron seleccionados para la lectura completa de sus respectivos resúmenes y éste; El 41,17\% fueron seleccionados para lectura completa. Los principales temas encontrados fueron la interferencia de la tecnología en la enseñanza de la disciplina de la anatomía, la comparación entre el método de enseñanza tradicional y la metodología asociada al uso de la realidad virtual y la simulación, y la motivación de los estudiantes sobre la posibilidad de utilizar las nuevas tecnologías en los procesos. de enseñanza-aprendizaje. Los resultados mostraron que existe una fuerte evidencia de que el uso de recursos tecnológicos estrecha las relaciones de estudiantes y docentes, ya que el proceso se vuelve más lúdico e integrador al tiempo que hace que el estudiante sea autónomo de su propia dirección en el aula, reduciendo el agotamiento inherente al estudio de la disciplina en su forma tradicional.

Palabras clave: Tecnología; Enseñanza; Anatomía; Bioingeniería.

\section{Introdução}

As práticas do ensino em saúde baseado em evidências na modernidade implicam na adoção do uso de tecnologia como forma de acompanhar as novas gerações de educandos, integrando-os ao melhoramento de práticas profissionais, bem como no que tange sua interface com modelos mais próximos à realidade (Myakava et al. 2021).

Vale ressaltar que bem como a resistência de considerável contingente de professores a aceitar o uso e prática em espaço de ensino-aprendizagem de ferramentas tecnológicas, Stepan et al (2017) reforça ainda que propriamente parte das unidades de ensino-aprendizagem que apresentam baixas disponibilidades de recursos, infraestruturas e variações de fatores internos, podem interferir consideravelmente em seu integrado processo de inovação ferramental e didático-tecnológico.

Ramos (2012) avalia que tais recursos tecnológicos educacionais, quando bem articulados, reforçam e viabilizam de maneira mais facilitada a direção da informação, tanto quanto sua partilha e elucidação do conhecimento por parte do aluno. Isto é, consonante na visão de Champaoski et al. (2018), a correta utilização de ferramentas educacionais de ordem tecnológicas, suprem uma demanda muito atual: a linguagem contemporânea.

A disciplina de anatomia é a base para a construção dos alicerces que fundamentam o domínio das ciências da saúde, sendo a mais tradicional disciplina dos cursos das áreas médicas. Com o surgimento da internet, o acesso ao conhecimento se 
tornou menos longínquo, uma vez que a associação de novas tecnologias interativas aproximara os discentes das novas possibilidades de assimilação de conteúdo (Lewis et al., 2014; Singh et al., 2021).

Aranda et al. (2016) avalia que o desafio proposto pelo ensino da disciplina de anatomia confronta-se com o professor responsável pelo processo pedagógico, isto porque, tradicionalmente os estudos em cima de tal área do conhecimento se pautam em peças cadavéricas para a abordagem exploratória do conteúdo à alunos de graduação, entretanto, na contemporaneidade o custo de manutenção de tais peças vem sendo calculado onerosamente, dificultando a qualidade do estudo uma vez que torna-se dispendioso o seu cuidado e também a forma de aquisição de tais elementos. Já para o ensino da disciplina de anatomia para o grau técnico do conhecimento da enfermagem, a presença de peças nos cursos é quase inexistente, e cabe aos alunos aprender com material puramente teórico disponibilizado por meio dos livros de anatomia, devendo o professor utilizar dos recursos mais variados o possível para alcançar a didática que visa o entendimento da matéria complexa ensinada. Neste sentido, o uso de peças impressas, além de fundamentar o aprendizado, baratearia o ensino a médio e longo prazo (Ovunc et al., 2021; Jiang et al., 2021).

O uso de softwares e programas de estudos possibilitou o enaltecimento de imagens que outrora eram encontrados somente em livros impressos, modelo consolidado e tradicional elencado às práticas pedagógicas de ensino. Ilustrações e representações gráficas na forma de imagem 3D estão cada vez mais próximas da realidade, permitindo a simulação muito semelhante à realidade factível (Pirri et al., 2021). Dessa forma, a elaboração do presente estudo justifica-se pela necessidade de avaliar as aplicações da tecnologia voltadas para o ensino da disciplina anatomia em cursos da área da saúde. A avaliação de um meio alternativo de ensino, tem objetivo de buscar evidências científicas a respeito das práticas tecnológicas empregadas na formação de alunos (Ghosh, 2015).

\section{Metodologia}

O trabalho foi desenvolvido com base em revisão de literatura (Gauch Jr, 2015; Estrela, 2018) nas bases de dados da Cochrane Library, LILACS e Mendeley, de 2008 a 2018. Para a realização da busca de palavras-chave foram utilizados os termos "tecnologia", "anatomia" e "ensino", bem como suas respectivas variações na língua inglesa "technology", "anatomy" e "education". Como critério de exclusão, foram adotadas as seguintes medidas: artigos publicados antes de 2008, que estivessem escritos em outras línguas que não fossem o inglês, espanhol ou português, e cujas temáticas não abordavam o uso de tecnologia para o ensino de anatomia. Foram encontrados 180 artigos após a busca nessas bases de dados, destes 17 artigos foram selecionados para leitura dos resumos e o restante foi descartado por não apresentar coerência com o propósito do presente estudo, sendo que a maior necessidade de eliminação foi devido ao envolvimento com temas relacionados à anatomia veterinária, à repetição de artigos, ao uso de impressora 3D, e também aqueles que não tratavam sobre tecnologia aplicada à educação. A metodologia aplicada para a revisão bibliográfica pode ser observada no Quadro 1, o qual relaciona a base de dados utilizada para pesquisa, a quantidade de resumos avaliados, os artigos selecionados para leitura crítica e avaliação integral, bem como a relação de títulos encontrados com o uso das palavras-chave selecionadas para a busca textual.

Quadro 1. Relação da metodologia aplicada.

\begin{tabular}{|c|c|c|c|}
\hline Base de Dados & $\begin{array}{c}\text { Resumos Avaliados } \\
\text { Integralmente }\end{array}$ & $\begin{array}{c}\text { Artigos Selecionados } \\
\text { para Avaliação }\end{array}$ & Títulos Encontrados \\
\hline Cochrane Library & 1 & 0 & 66 \\
\hline LILACS & 5 & 4 & 90 \\
\hline Mendeley & 4 & 0 & 7 \\
\hline Outros & 7 & 3 & 180 \\
\hline Total & 17 & 7 & 78 \\
\hline
\end{tabular}

Fonte: Autores (2021). 


\section{Resultados e Discussão}

Dos 17 artigos selecionados para leitura dos resumos, somente 7 foram avaliados integralmente. A pesquisa realizada a partir do uso das palavras-chave selecionadas evidenciou que há certa variedade de linhas de pesquisa envolvendo o uso de tecnologia direcionada para a educação em saúde. O Quadro 2 relaciona os resultados encontrados, no qual é possível observar a coluna "Tema" com o assunto tratado pelo artigo, "Busca" com a plataforma onde se encontrou e "Autores" com as referências mencionadas.

Quadro 2. Associação de busca do tema com a plataforma e autores.

\begin{tabular}{|l|l|l|}
\hline Tema & Autores & Busca \\
\hline Interferência da tecnologia no aprendizado. & Trotta e Spinillo, 2014. & Outros \\
\hline Realidade aumentada para o ensino da anatomia. & Fernandes et al, 2013. & Outros \\
\hline $\begin{array}{l}\text { Comparação do ensino da anatomia do método clássico com o } \\
\text { moderno. }\end{array}$ & Silva et al, 2017. & LILACS \\
\hline $\begin{array}{l}\text { Associação da tecnologia para ensino de anatomia e fisiologia com } \\
\text { foco em aferição de sinais vitais em enfermagem. }\end{array}$ & Góes et al, 2015. & LILACS \\
\hline $\begin{array}{l}\text { Validação de software para ensino prático de anatomia com foco } \\
\text { em sistema venoso periférico em enfermagem. }\end{array}$ & Gardioli et al, 2018. & LILACS \\
\hline $\begin{array}{l}\text { Avaliação do impacto em alunos de medicina do uso de } \\
\text { simuladores virtuais para o processo de ensino de anatomia. }\end{array}$ & Hecht-Lopez et al, 2018. & LILACS \\
\hline $\begin{array}{l}\text { Construção de aplicações educacionais utilizando a tecnologia do } \\
\text { metaverso para aulas de anatomia. }\end{array}$ & Massaro et al, 2011. & Outros \\
\hline
\end{tabular}

Fonte: Autores (2021).

Góes et al. (2015), avaliou o uso de tecnologia voltada ao ensino de anatomia e fisiologia em curso profissionalizante de enfermagem com foco em aferição de sinais vitais ressaltando que o uso de tecnologias contribui para a superação dos limites do processo pedagógico de ensino para o aluno, de forma que tal facilidade não implica na substituição do professor em sala de aula, mas na melhora da perspectiva profissional.

No contexto da pesquisa, foram avaliados 21 estudantes dentre os 45 selecionados no total, dentre os quais, $57 \%$ concordaram com a recomendação do uso da tecnologia para o ensino da enfermagem ser positivo, e 52\% concordam fortemente com o fato de a tecnologia auxiliar no processo de aprendizado do aluno.

Para Gadioli et al. (2018), o uso da tecnologia auxiliou uma alternativa na prática educacional de anatomia e fisiologia com foco de aprimoramento da semiologia do sistema venoso periférico. Em seu estudo, 20 estudantes de enfermagem foram avaliados e 10 profissionais já formados comparando-se o método de ensino tradicional ao método com tecnologia associada ao ensino prático da disciplina.

Do total de participantes da pesquisa, 95\% considerou que o uso de tecnologia direcionado ao ensino da anatomia tornava a aprendizagem mais fácil, $85 \%$ relatou não apresentar dificuldades em associar a tecnologia com a disciplina e $95 \%$ classificou o método como motivador para os estudos.

Hecht e Larrazabal (2018), aponta em seu estudo que o uso de tecnologia para o ensino da disciplina de anatomia humana proporciona melhora do desempenho dos estudantes no que cerne ao aprendizado. Em seu estudo, $96 \%$ dos avaliados concordou totalmente que as plataformas de ensino digital e os recursos de tecnologia são úteis para o ambiente acadêmico, contrapondo-se a um resultado de aproximadamente $4 \%$ que apontou o uso da tecnologia como algo indiferente ao seu estudo. 
Para os pesquisadores Fernandes et al. (2011), a tecnologia aproxima a realidade do aluno ao passo que proporciona o avanço da exploração do ensino através das interfaces alcançadas com a imersão do usuário em ambientes elucidados pela interação.

Dessa forma, o estudo aponta como vantagens o uso da realidade aumentada para fins educacionais, a motivação dos estudantes de uma forma geral, o suporte à visualização de elementos que estão inacessíveis, à exploração de domínios periféricos, além de possibilitar que o discente se torne ativo no processo de aprendizagem propiciado pela visualização e ampliação da realidade.

Para Massaro et al. (2011), a proposta do desenvolvimento de uma ferramenta tecnológica que auxilie tanto o professor quanto o aluno no processo do ensino-aprendizagem da disciplina de anatomia pode aprimorar a didática, uma vez que é possível desenvolver a percepção tridimensional dos elementos estudados.

Já os pesquisadores Trotta e Spinillo (2017), apontam que o ensino da anatomia mediado por tecnologia permite ao aluno armazenar dados em sua memória através da visualização espacial e que a interatividade é o ponto principal desse processo. Assim, o ensino torna-se dinâmico e clinicamente interativo, possibilitando repensar os modelos tradicionais da troca de informação, além do que, essa associação reduz o tempo e o custo com a dissecação de cadáveres reais ao passo que estruturas anatômicas podem ser projetadas em realidade virtual para aprendizagem.

Para Silva et al. (2017), as novas tecnologias de inovação e informação corroboram para a aproximação entre professor e aluno, com a justificativa de que no modelo de ensino tradicional é comum que o discente se torne disperso no transcorrer das aulas, sendo esse um processo natural e fisiológico do corpo que está contemplado no método de aprendizagem passivo, contrapondo-se a isso, o uso de tecnologia e softwares propiciam um aprendizado ativo uma vez que obtém praticamente o foco total do aluno ao provocar a imersão do conteúdo de aprendizagem conferindo autonomia para o mesmo.

\section{Conclusão}

Os resultados encontrados evidenciam que o uso de recursos tecnológicos para o ensino da anatomia apresenta aspectos favoráveis para a aprendizagem dos alunos, uma vez que os mesmos adquirem autonomia para conduzir seu estudo através da linha de raciocínio que melhor se adapta à sua forma de entendimento, fato este que aponta para a motivação dos mesmos diante de novas possibilidades de interação. Dessa forma, o presente artigo cumpriu com os objetivos propostos justificado pelas evidências encontradas através de publicações em revistas e anais de congresso de ferramentas tecnológicas inovadoras que fomentam a diversificação da metodologia de ensino para cursos da área da saúde.

\section{Referências}

Aranda, W. D., Daré, L. R., \& Dias, D. V. (2016). A inserção do atlas 3D no processo de ensino e aprendizagem em anatomia humana. Semantic Scholar. 1, 01-03.

Champaoski, E. B., Mendes, A. A. P., \& Silva, W. (2018). Percepção docente acerca das tecnologias digitais no cotidiano escolar. In: XII Congresso Nacional de Educação. Anais [...]. Fortaleza: XII EDUCERE. 21461-21470.

Estrela, C. (2018). Metodologia Científica: Ciência, Ensino, Pesquisa. Editora Artes Médicas.

Fernandes, F. G, Oliveira, L. C, Barbosa, A. J, Moura, C. C. O., Rodrigues, M. L., \& Vita, S. S. B. V. (2013). Ensino da anatomia do corpo humano usando a realidade aumentada móvel. Conferência de Estudos em Engenharia Elétrica. 1-6.

Gadioli, B., Fulquini, F. L., Kusumota, L., Gimenes, F. R. E., \& Carvalho, E. C. (2018). Construction and validation of a virtual learning object for the teaching of peripheral venous vascular semiology. Escola Anna Nery. 22(4):1-8.

Gauch Jr, H. (2015). Scientific Method in Practice. Cambridge: Cambridge University Press. 10.1017/CBO9780511815034

Ghosh, S. K. (2015). Evolution of Illustrations in Anatomy: A Study from the Classical Period in Europe to Modern Times. Anatomical Sciences Education.8(2):175-188. 
Góes, F. S. N. de, Camargo, R. A. A. de, Fonseca, L. M. M., Oliveira, G. F. de, Hara, C. Y. N., Felipe, H. R., \& Caldas, N. B. (2015). Avaliação de tecnologia digital educacional - sinais vitais e anatomia - por estudantes da educação profissionalizante em enfermagem. Rev Min Enferm.19(2):37-43.

Hecht, L. P., \& Larrazabal, M. A. (2018). Uso de nuevos recursos tecnológicos en la docencia de un curso de anatomía con orientación clínica para estudiantes de medicina. Int. J. Morphol. 36(3):821-828.

Jiang, M., Coles-Black, J., Chen, G., Alexander, M., Chuen, J., \& Hardidge, A. (2021). 3D Printed Patient-Specific Complex Hip Arthroplasty Models Streamline the Preoperative Surgical Workflow: A Pilot Study. Frontiers in surgery, 8, 687379. https://doi.org/10.3389/fsurg.2021.687379

Lewis, T. L., Burnett, B., Tunstall, R., \& Abrahams, P. (2014). Complementing anatomy education using three-dimensional anatomy mobile software applications on tablet computers. Clinical Anatomy.27(3):313-320.

Massaro, G., Mantovani, A. M., \& Rodrigues, M. S. (2011). Aplicações educacionais em 3d para os processos de ensino e aprendizagem da área de anatomia no second life. Renote - Novas Tecnologias na Educação. 9(2):1-10.

Myakava, L., Santos, M., \& Püschel, V. (2021). Knowledge, skills, and attitudes of nursing students on evidence-based practice. Revista da Escola de Enfermagem da USP, 55, e20200428. https://doi.org/10.1590/1980-220X-REEUSP-2020-0428

Ovunc, S. S., Yassin, M., Chae, R., Abla, A., \& Rodriguez Rubio, R. (2021). Constructing an Individualized Middle Cerebral Artery Model Using 3D Printing and Hydrogel for Bypass Training. Cureus, 13(7), e16749. https://doi.org/10.7759/cureus.16749

Pirri, C., Stecco, C., Porzionato, A., Boscolo-Berto, R., Fortelny, R. H., Macchi, V., Konschake, M., Merigliano, S., \& De Caro, R. (2021). Forensic Implications of Anatomical Education and Surgical Training With Cadavers. Frontiers in surgery, 8, 641581. https://doi.org/10.3389/fsurg.2021.641581

Ramos, M. R. V. (2012). O uso da tecnologia em sala de aula. Revista Eletrônica LENPES-PIBID de Ciências Sociais, 1, 01-15.

Singh, P., Phuyal, N., Khadka, S., \& Gurung, M. (2021). Knowledge of Medical Students and Faculties of a Medical College Towards Human Body and Organ Donation: A Descriptive Cross-sectional Study. JNMA; journal of the Nepal Medical Association, 59(234), 141-145. https://doi.org/10.31729/jnma.6200

Silva, A. R., Valério, M. M. C., Neto, P. A. M., \& Campos Filho, A. S. (2017). Anatomia digital: um ambiente virtual de apoio ao processo ensinoaprendizagem. VI Congresso Brasileiro de Informática na Educação. Anais do XXVIII - Simpósio Brasileiro de Informática na Educação. Recife, p.745-755.

Stepan, K., Zeiger, J., Hanchuk, S., Del Signore, A., Shrivastara, R., Govindaraj, S., \& Iloreta, A. (2017). Immersive virtual reality as a teaching tool for neuroanatomy. International Forum of Allergy and Rhinology.7(10):1006-1013.

Trotta, T., \& Spinillo, C. G. (2017). Tecnologias no aprendizado da anatomia humana: possíveis contribuições para o ensino da medicina. Brazilian Journal of Information Design.11(1):1-20. 